\title{
Analysis of activin/TGFB-signaling modulators within the normal and dysfunctional adult human testis reveals evidence of altered signaling capacity in a subset of seminomas
}

\author{
Vinali L Dias ${ }^{1}$, Ewa Rajpert-De Meyts ${ }^{4}$, Robert McLachlan ${ }^{5}$ and Kate Lakoski Loveland ${ }^{2,3}$ \\ ${ }^{1}$ Monash Institute of Medical Research, Monash University, Clayton, Victoria 3168, Australia, ${ }^{2}$ Departments of \\ Biochemistry and Molecular Biology, Monash University, Building 77 Level 1, Wellington Road, Clayton, \\ Victoria 3800, Australia, ${ }^{3}$ Australian Research Council Centre of Excellence in Biotechnology and Development, \\ Australia, ${ }^{4}$ University Department of Growth and Reproduction, Rigshospitalet, DK-2100, Copenhagen, Denmark \\ and ${ }^{5}$ Prince Henry's Institute of Medical Research, Clayton, Victoria 3168, Australia
}

Correspondence should be addressed to K L Loveland at School of Biomedical Sciences, Building 77, Level 1, Wellington Road, Monash University, Clayton, Victoria 3800, Australia; Email: kate.loveland@med.monash.edu.au

\begin{abstract}
Activin is a pleiotropic growth factor belonging to the transforming growth factor- $\beta$ (TGFB) superfamily of signaling molecules. Regulated activin signaling is known to influence several steps in rodent male gamete differentiation. TGFB ligand isoforms, TGFB1-B3, also influence germ cell survival in the rodent testis at the onset of spermatogenesis and around the time of puberty. Given the importance of regulated activin and TGFB signaling in testis development and function, we sought to investigate the cellular production sites of activin/TGFB-signaling modulators in normal and dysfunctional adult human testes samples. Signaling transducers phosphorylated SMAD2/3, and signaling modulators SMAD6, MAN-1, inhibin $\alpha$ (INHA), and $\beta$-glycan were detected in Bouins fixed, paraffin-embedded adult human testis sections using immunohistochemistry. Additional samples examined were from testicular cancer patients and from normal men subjected to gonadotropin suppression with androgen-based contraceptives. Our findings identify distinct differences between normal and gonadotropin-deprived human testis in the expression and cellular localization of activin/TGFB-signaling modulators. The presence of a nuclear phosphorylated SMAD2/3 signal in all analyzed seminoma specimens indicated active activin/TGFB signaling. Moreover, a subset of seminoma specimens exhibited selective enhanced expression of $\beta$-glycan (4 out of 28 seminoma tumors), INHA (6 out of 28), and MAN-1 (6 out of 28), highlighting potential functional differences between individual tumors in their capacity to regulate activin/TGFB signaling. Within the heterogenous nonseminomas, expression of signaling modulators was variable and reflected the degree of somatic differentiation. Thus, synthesis of activin and TGFB-signaling modulators may be affected by spermatogenic disruption and altered hormone levels in the testis.
\end{abstract}

Reproduction (2009) 138 801-811

\section{Introduction}

The transforming growth factor- $\beta$ (TGFB) superfamily of pleiotropic growth factors exert a broad range of effects on the differentiation, proliferation, and function of numerous cell types, including those in the mammalian testis (Loveland \& Robertson 2005). Among these, signaling by the activin ligand in particular has been shown to play pivotal roles in the development and function of both germ and Sertoli cells in the juvenile and adult rodent testis (Mather et al. 1990, Matzuk et al. 1992, 1995, Boitani et al. 1995, Brown et al. 2000, Meehan et al. 2000, Fragale et al. 2001, reviewed in Itman et al. (2006)). All three TGFB ligand isoforms, TGFB1-B3, also act in vitro to regulate rodent gonocyte, pubertal spermatogonia, and spermatocyte numbers by inducing germ cell apoptosis (Olaso et al. 1998,
Konrad et al. 2006). Expression of two activin and TGFB modulators at distinct stages of rodent spermatogenesis has been previously documented, indicating the presence of regulated TGFB superfamily signaling during germ cell development (Meehan et al. 2000, Loveland et al. 2003). In the newborn rat testis, the membranebound pseudoreceptor Bambi mRNA and activin antagonist follistatin mRNA and protein are up-regulated in gonocytes at day 3 of postnatal testis development, around the time when gonocytes are differentiating into spermatogonia, indicating that downregulation of activin signaling is required for germ cell maturation in rodents (Meehan et al. 2000, Loveland et al. 2003).

TGFB superfamily signaling is initiated at the cell surface by ligand interaction with type I (ACVR1B and ACVR1C for activin, and ACVRL1 and TGFBR1 for TGFB 
isoforms) and type II (ActRIIA, ActRIIB, and TGFBR2) transmembrane receptors, each with intrinsic serine/ threonine kinase activity (Chang et al. 2002, Shi \& Massague 2003, Mazerbourg et al. 2005, Lin et al. 2006). Ligand binding enables formation of a heterotetrameric receptor complex between type I and type II receptors, which leads to the selective phosphorylation and activation of the activin/TGFB-specific intracellular signal transducers, SMAD2 and SMAD3. These interact with the common SMAD, SMAD4, and translocate into the nucleus to initiate transcription of activin/TGFB response genes (Chang et al. 2002, Shi \& Massague 2003, Lin et al. 2006).

Regulation of activin and TGFB signaling has been identified at each level of their signaling cascades (Harrison et al. 2004, 2005). At the cell surface, inhibin and its co-receptor $\beta$-glycan interfere with signaling by competing with activin for binding to type II activin receptors (Lewis et al. 2000, Esparza-Lopez et al. 2001). Expression of $\beta$-glycan also confers sensitivity to all three TGFB ligand isoforms and to the bone morphogenetic protein (BMP) ligands, BMP2, 4, 7 and GDF5, by acting as a co-receptor to promote ligand interaction with serine/threonine kinase receptors and facilitate signal transduction (Lopez-Casillas et al. 1993, Kirkbride et al. 2008). Inside the cell, the inhibitory SMADs, SMAD6 and SMAD7, prevent activation and signal propagation by receptor-regulated SMAD proteins in the cytoplasm and negatively regulate gene transcription in the nucleus. Among the inhibitory SMADs, SMAD6 preferentially inhibits BMP signaling and only weakly inhibits TGFB/activin signaling, whereas SMAD7 ubiquitously inhibits TGFB, activin, and BMP signaling (Itoh et al. 1998, Ishisaki et al. 1999, Hanyu et al. 2001, Goto et al. 2007). Moreover, expression of inhibitory SMADs is induced by TGFB superfamily signaling, creating a negative feedback loop to modulate the magnitude and duration of TGFB/activin and BMP signaling (Nagarajan et al. 1999, Denissova et al. 2000, Ishida et al. 2000). At the inner nuclear membrane, MAN-1 interacts with phosphorylated SMAD2/3 and target DNA sequences to inhibit transcriptional activation of activin/TGFB response genes (Imamura et al. 1997, Bai \& Cao 2002, Lin et al. 2005, Pan et al. 2005, Caputo et al. 2006).

The function of activin and TGFB signaling and the mechanisms by which it is regulated in the human testis have not yet been defined. Cell-specific expression analysis of activin antagonists in the normal and dysfunctional human testis is largely limited to the inhibin $\alpha$-subunit (INHA), which is produced by Sertoli cells within the seminiferous epithelium and by interstitial Leydig cells in fetal and adult testes. Both INHA and $\beta$-glycan are produced by Leydig cells in the fetal testis (Vliegen et al. 1993, Majdic et al. 1997, Roberts 1997, Andersson et al. 1998, Anderson et al. 2002, Marchetti et al. 2003), indicating regulated TGFB superfamily signaling features during normal human fetal testis development and that gonocytes do not make these proteins. In addition, RNAse protection assays and immunohistochemical analysis have identified the INHA subunit mRNA and protein in a subset of testicular germ cell tumors (TGCTs), indicating that individual tumors differ in their responses to activin and TGFB signaling (van Schaik et al. 1997, Cobellis et al. 2001).

Testicular cancer accounts for $1 \%$ of neoplasms in men and is the most common malignancy in males aged between 15-35 years (Bergstrom et al. 1996). TGCTs arise from a preinvasive precursor cell called carcinoma in situ (CIS), first described by Skakkebaek (1972) as atypical spermatogonia in testicular biopsies of patients who subsequently developed testicular cancer. CIS cells are thought to originate during fetal life due to an arrest in the differentiation program of fetal gonocytes or primordial germ cells (Rajpert-de Meyts \& Hoei-Hansen 2007). Comparative analyzes of protein and RNA expression levels underpin this hypothesis, as seen by shared production of POU5F1 (OCT3/4), NANOG, AP2 $\gamma$, KIT and PDPN (podoplanin/M2A) between CIS cells and human gonocytes (Sonne et al. 2006, Rajpert-de Meyts \& Hoei-Hansen 2007). Later in life, usually after puberty, CIS cells transform to overt germ cell tumors, either seminomas or nonseminomas, presumably in response to the changing hormonal environment at this time. Seminomatous tumors proliferate along the germ cell lineage, while nonseminomas retain embryonic features such as embryonal carcinoma cells that can differentiate into somatic (teratomas) and extraembryonic (yolk-sac tumors and choriocarcinomas) lineages.

Our previous findings identified spermatogonia and Sertoli cells as the predominant targets of activin signaling in the adult human testis, based on the selective production of three activin receptor subunits ALK2, ACVR1B and ActRIIB in these cells (Dias et al. 2008). We also described upregulation of a fourth activin receptor subunit, ActRIIA, as preinvasive CIS cells progress to malignant seminomas, indicating the capacity for aberrant activin signaling to occur in germ cell neoplasms (Dias et al. 2008). Enhanced production of ActRIIA relative to normal testes was also detected in gonadotropin-deprived human testis samples, obtained following spermatogenic suppression in men treated with testosterone and depot progestin (McLachlan et al. 2002, Dias et al. 2008). The increased synthesis of ActRIIA in both seminomas and gonadotropin-deprived samples suggests the potential for activin signaling to be affected by changes in hormone levels. This could result in altered signaling within specific cells of the dysfunctional human testis by activin/TGFBs and other TGFB superfamily ligands, BMPs and Nodal, which also utilize ActRIIA for signal transduction.

In this study, we set out to define the cellular sites of production of activin and TGFB-signaling modulators in the normal, gonadotropin-deprived, and 
neoplastic adult human testis. These include the activin/TGFB-signaling transducers, phosphorylated SMAD2/3 and activin antagonists, INHA, $\beta$-glycan, SMAD6, and MAN-1. Phosphorylation of SMAD2/3 is an indication of activin/TGFB-signaling activity (reviewed in Shi \& Massague 2003), INHA and $\beta$-glycan are activin-signaling antagonists normally restricted to somatic cells of the testis (Vliegen et al. 1993, Majdic et al. 1997, Andersson et al. 1998, Anderson et al. 2002, Marchetti et al. 2003), and SMAD6 and MAN-1 have yet to be assessed in the contexts of normal and dysfunctional adult human testis. Our findings demonstrate a nuclear phosphorylated SMAD2/3 signal in all seminoma tumors, indicating active activin/TGFB signaling. Our results also identify INHA, $\beta$-glycan, and MAN- 1 to be present in selected seminoma tumors, indicating that these factors may limit the bioavailability of activin and TGFBs in a subset of testis tumors.

\section{Results}

\section{Western blot verification of anti-MAN-1 antibody}

Target recognition by the goat polyclonal anti-MAN-1 (N-20; Santa Cruz Biotechnology, Santa Cruz, CA, USA) was determined by western blot analysis on human NTERA cell lysates. A single band of the expected size (82 $\mathrm{kDa})$ was detected, indicating selective detection of the MAN-1 protein (Fig. 1).

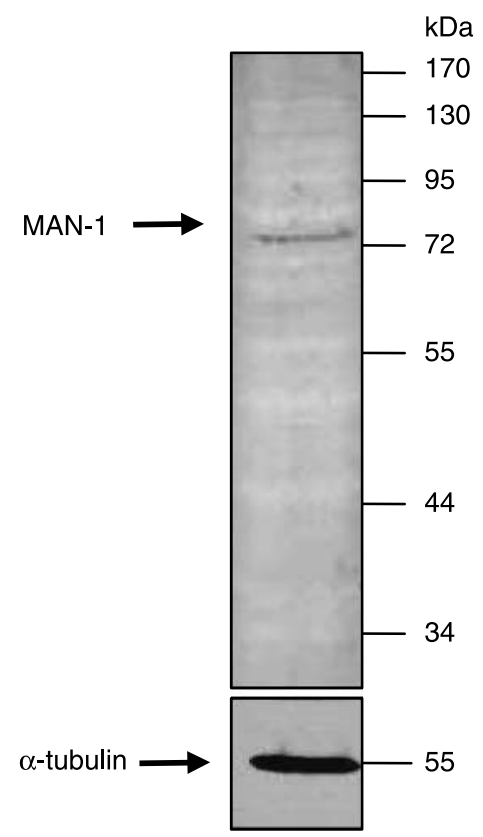

Figure 1 Western blot validation of anti-MAN-1. A single band of the expected band size of $82 \mathrm{kDa}$ for MAN-1 was detected in human NTERA cell lysates. Protein loading was indicated by blotting for $\alpha$-tubulin.
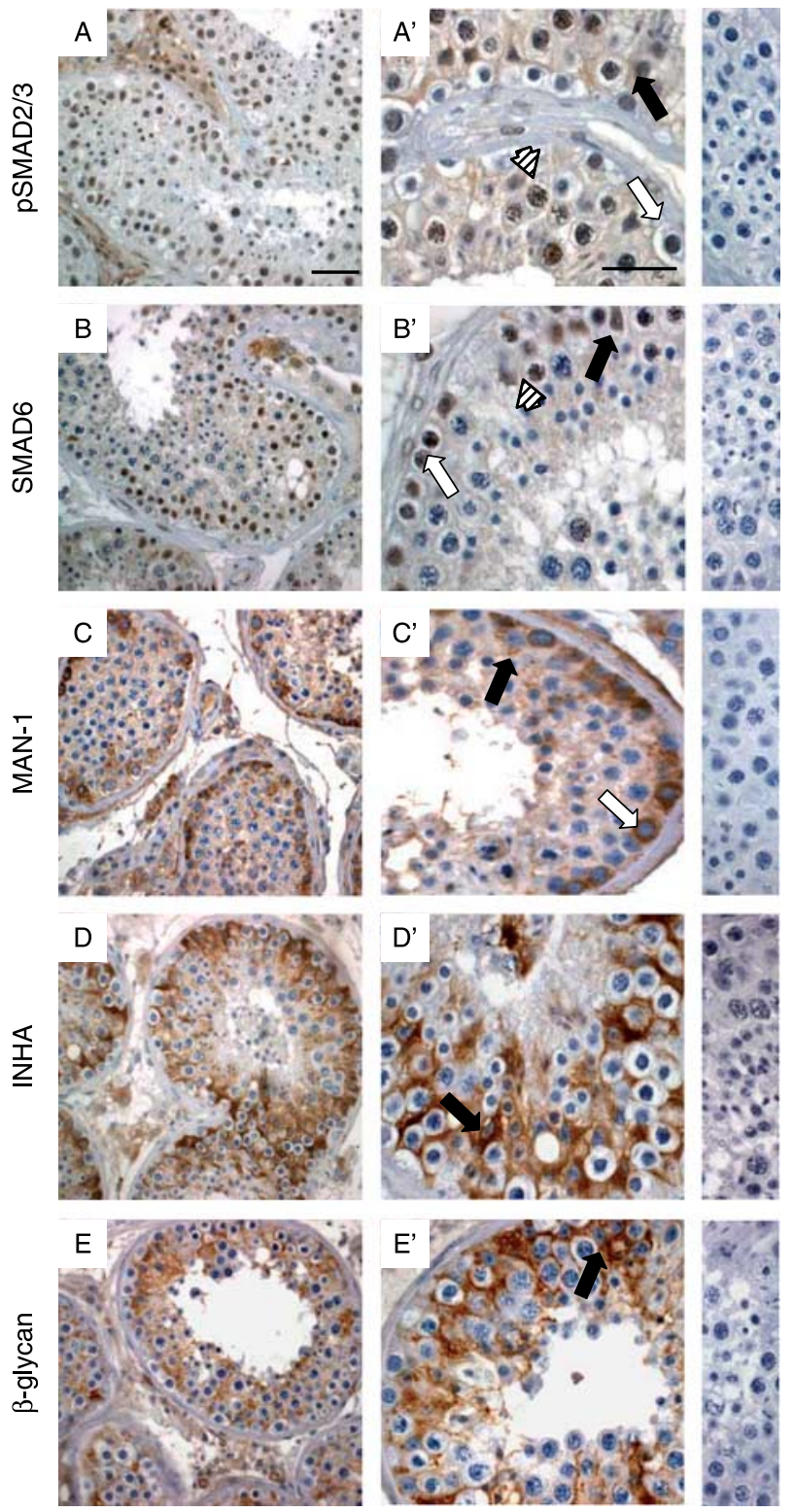

Figure 2 Cellular localization of activin and TGFB-signaling modulator proteins within normal human testis. Low magnification (A-E) and high magnification $\left(A^{\prime}-E^{\prime}\right)$ images of immunohistochemical detection for activin/TGFB signal transducing phosphorylated SMAD2/3 (A and $A^{\prime}$ ), and activin/TGFB regulators SMAD6 (B and $\left.\mathrm{B}^{\prime}\right), \mathrm{MAN}-1$ ( $\mathrm{C}$ and $\left.\mathrm{C}^{\prime}\right)$, INHA ( $D$ and $\left.D^{\prime}\right)$, and $\beta$-glycan ( $E$ and $E^{\prime}$ ) in adult human testis sections. All antigens were detected in association with Sertoli cells. In addition, phosphorylated SMAD2/3 signal was present in the nucleus of all germ cell types, SMAD6 in the nucleus of spermatogonia and spermatocytes, and MAN-1 at the nuclear periphery and cytoplasm of spermatogonia and occasional spermatocytes and round spermatids. Right-hand panels represent negative control sections lacking each primary antibody. White arrow, spermatogonial cell staining; hatched arrowhead, spermatocyte staining; black arrow, Sertoli cell staining. pSMAD2/3, phosphorylated SMAD2/3. Scale bars in panel A and panel $A^{\prime}$ are representative of panels $A-E$ and $A^{\prime}-E^{\prime}$ respectively indicating $50 \mu \mathrm{m}$. 


\section{Activin and TGFB-signaling modulators within the normal adult human testis}

Within all sections $(n=3)$ of phenotypically normal adult human testis, immunostaining for activin/TGFB-signaling modulators was consistently detected in Sertoli cells. Specifically, phosphorylated SMAD2/3 was detected within Sertoli cell nuclei, SMAD6 within Sertoli cell nuclei and cytoplasm, MAN-1 within the cytoplasm and nuclear periphery of occasional Sertoli cells, and INHA and $\beta$-glycan within the Sertoli cell cytoplasm (Fig. 2). Leydig cells exhibited immunostaining for all antigens. Within the seminiferous tubules, phosphorylated SMAD2/3 was localized to the nucleus of all germ cell types, SMAD6 to the nucleus of spermatogonia and spermatocytes, and MAN-1 to the nuclear periphery and cytoplasm of most spermatogonia and occasional spermatocytes and round spermatids (Fig. 2 and Table 1). INHA and $\beta$-glycan were not detected in association with germ cells. These results identify Sertoli cells and spermatogonia as the common sites of production of both transducers and modulators of activin and TGFB signaling within the seminiferous epithelium.

\section{Activin and TGFB-signaling modulators within gonadotropin-deprived testis}

To examine the effects of gonadotropin and intratesticular testosterone suppression on the expression of activin/TGFB-signaling modulators, human testis sections were obtained from a previous study investigating spermatogenic failure induced by androgen-based contraceptive treatments (McLachlan et al. 2002). As the immunostaining patterns following 12 weeks of testosterone or 12 weeks of testosterone plus depot medroxyprogesterone acetate (DMPA) treatment were indistinguishable for all antigens, only staining of the former is shown (Fig. 3). Immunostaining for phosphorylated SMAD2/3 exhibited a nuclear signal in all germ cell types and Sertoli cells in all testosterone or testosterone plus DMPA-treated samples (Fig. 3 and Table 1). Interestingly, the intensity of this nuclear signal was markedly stronger in all 12-week testosterone or testosterone plus DMPA-treated samples $(n=4$ for each treatment group) than in untreated testis samples from the same study $(n=3)$. Testis samples from men subjected to testosterone or testosterone plus DMPA treatment for 12 weeks demonstrated SMAD6 protein in the nucleus and cytoplasm of Sertoli cells, spermatogonia, and spermatocytes (Fig. 3 and Table 1). Relative to staining in the normal untreated testis samples, SMAD6 exhibited a distinct cytoplasmic signal in spermatocytes within gonadotropin-deprived samples (Fig. 3). Immunostaining for MAN-1 labeled the nuclear periphery and cytoplasm of most spermatogonia, Sertoli cells, and occasional spermatocytes in untreated and 12-week gonadotropin-deprived testis samples (Fig. 3 and Table 1). In men treated for 12 weeks with testosterone or testosterone plus DMPA, strong immunostaining for INHA and $\beta$-glycan was detected in association with Sertoli cells (Fig. 3 and Table 1). In contrast to the normal untreated adult human testis specimens $(n=3), \beta$-glycan protein was also localized to round spermatids in all sections from men subjected to 12 weeks of gonadotropin suppression ( $n=4$ for each treatment group, Fig. 3). These results identify specific changes in the localization and expression patterns of activin/TGFB-signaling modulators in gonadotropin-deprived human testes samples, providing evidence that changes in activin and TGFBsignaling regulation occur in response to spermatogenic suppression.

Table 1 Summary of activin and transforming growth factor- $\beta$ (TGFB)-signaling modulator protein immunostaining levels in the normal adult human testes, testicular carcinomas, and gonadotropin-deprived human testes.

\begin{tabular}{|c|c|c|c|c|c|c|c|}
\hline & \multirow[b]{2}{*}{$\begin{array}{c}\text { Normal } \\
\text { (untreated) testes }\end{array}$} & \multicolumn{2}{|c|}{ Gonadotropin-deprived human testes } & \multicolumn{4}{|c|}{ Testicular carcinomas } \\
\hline & & $\begin{array}{l}\text { Testosterone } \\
12 \text { weeks }\end{array}$ & $\begin{array}{l}\text { Testosterone }+ \\
\text { DMPA } 12 \text { weeks }\end{array}$ & $\begin{array}{l}\text { Normal tubules } \\
\text { in CIS }\end{array}$ & $\mathrm{CIS}$ & Seminoma & $\begin{array}{c}\text { Non } \\
\text { seminoma }\end{array}$ \\
\hline Sample number & $(n=3)$ & $(n=4)$ & $(n=4)$ & $(n=7)^{\mathrm{a}}$ & $(n=14)^{\mathrm{a}}$ & $(n=28)^{\mathrm{b}}$ & $(n=9)^{\mathrm{a}}$ \\
\hline Staining intensity & $+++^{c}$ & +++ & +++ & $+++^{c}$ & +++ & See below & +++ \\
\hline $\begin{array}{l}\text { Phosphorylated } \\
\text { SMAD2/3 }\end{array}$ & Sc, SG, SC, R & Sc, SG, SC, R & Sc, SG, SC, R & Sc, SG, SC, R & $\mathrm{Sc}, \mathrm{CIS}$ & $+++{ }^{d}$ Sem & V \\
\hline SMAD6 & Sc, SG, SC & Sc, SG, SC & Sc, SG, SC & Sc, SG, SC & Sc, CIS & +++ Sem & V \\
\hline MAN-1 & $\begin{array}{l}\text { Sc, SG, and } \\
\text { some SC }\end{array}$ & $\begin{array}{l}\text { Sc, SG, and } \\
\text { some SC }\end{array}$ & $\begin{array}{l}\text { Sc, SG, and } \\
\text { some SC }\end{array}$ & $\begin{array}{l}\text { Sc, SG, and } \\
\text { some SC }\end{array}$ & Sc, CIS & $+1-{ }^{\mathrm{e}} \mathrm{Sem}$ & V \\
\hline INHA & Sc & Sc & $\mathrm{SC}$ & Sc & Sc & $+1-{ }^{\mathrm{e}}$ Sem & V \\
\hline$\beta$-Glycan & Sc & Sc, $\mathrm{R}$ & Sc, $\mathrm{R}$ & Sc & Sc & $+/-{ }^{f}$ Sem & V \\
\hline
\end{tabular}

Samples scored according to subjective, semiquantitative analysis. +++ , strong staining intensity; ++ , moderate staining intensity; + , low staining intensity; - , no staining. Testosterone; DMPA, depot medroxyprogesterone acetate; SG, spermatogonia; SC, spermatocyte; R, round spermatid; Sc, Sertoli cell; Sem, seminoma cell; V, various cell types; N/A, not tested.

${ }^{a} n=4$ for SMAD6; ${ }^{b} n=6$ for SMAD6; ${ }^{c}++$ signal for phosphorylated SMAD2/3; ${ }^{d}$ Three out of 28 samples with $+/-$ signal; ${ }^{\text {e }}$ Six out of 28 samples with ++ signal; ${ }^{f}$ Four out of 28 samples with +++ signal. 


\section{Activin and TGFB-signaling modulators within testicular neoplasms}

Expression of activin/TGFB-signaling modulators was examined within a range of testicular cancer specimens consisting of CIS, seminomas, and nonseminomas. Immunohistochemistry signals in tubules with retained complete spermatogenesis within CIS specimens provided controls for comparison with normal testes samples as shown in Fig. 4. The premalignant CIS cells exhibited nuclear localization of phosphorylated SMAD2/3 $(n=14)$ and SMAD6 $(n=4)$, lacked INHA $(n=14)$ and $\beta$-glycan $(n=14)$ proteins, and exhibited a cytoplasmic signal for MAN-1 $(n=14)$ (Fig. 4 and Table 1). All five antigens were detected within Sertoli cells of CIS tubules. In seminoma tumors, SMAD6 $(n=6)$ and phosphorylated SMAD2/3 $(n=28)$ proteins localized
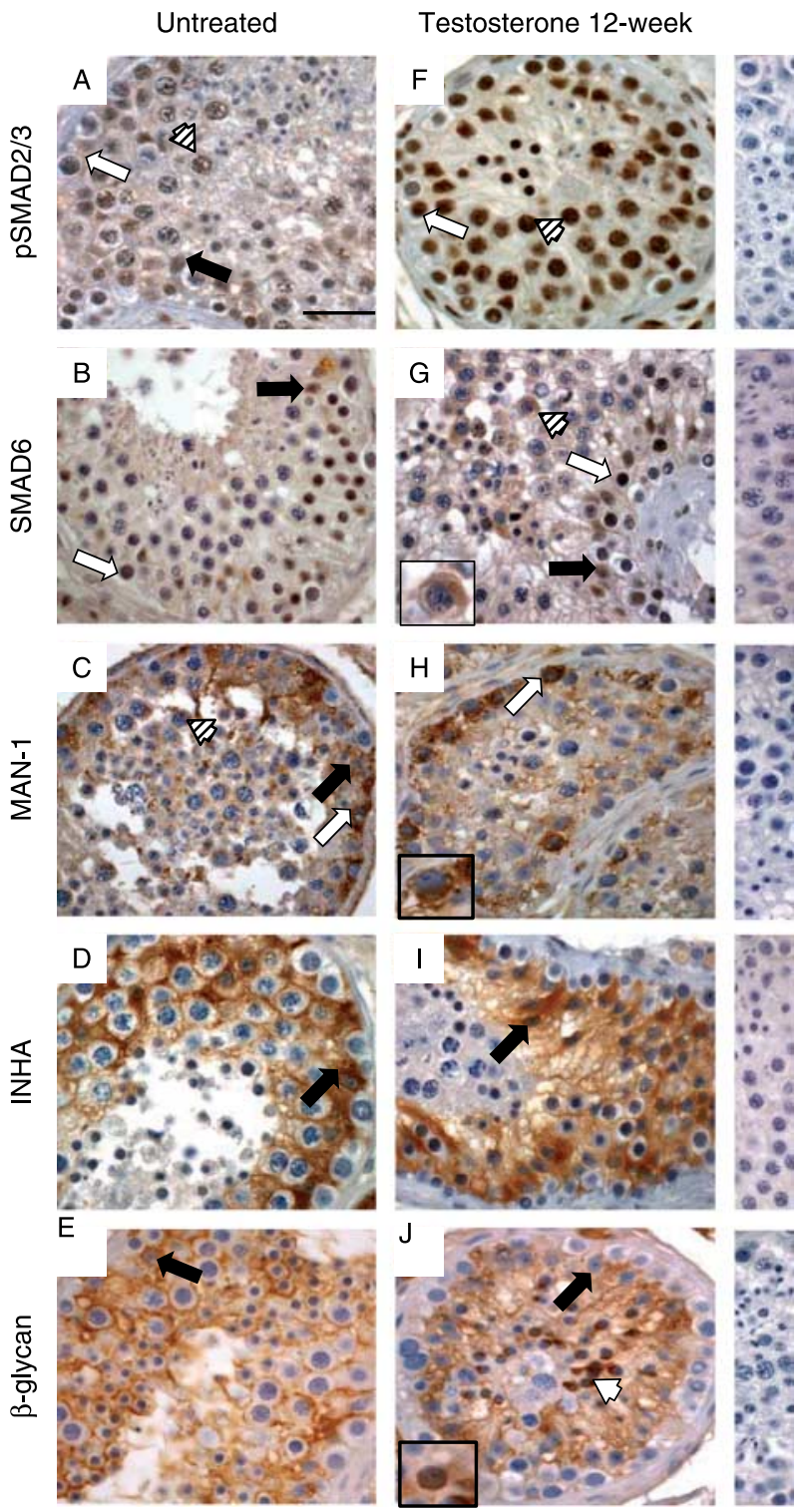

to the nuclei of seminoma cells, although 3 out of the 28 tumors tested showed a much weaker phosphorylated SMAD2/3 nuclear signal (Fig. 5A and Table 1). Similarly, MAN-1 $(n=28)$, INHA $(n=28)$, and $\beta$-glycan $(n=28)$ expression was heterogeneous, with a subset of tumors exhibiting a comparatively strong signal in seminoma cells across majority of the section for MAN-1 (6 out of 28 seminoma tumors; Fig. 5h), INHA ( 6 out of 28; Fig. 5D), and $\beta$-glycan ( 4 out of 28 ; Fig. 5E; Table 1). Among the subset of tumors that displayed heterogeneous staining for phosphorylated SMAD2/3, MAN-1, INHA, and $\beta$-glycan, three individual seminoma tumors displayed intriguing correlations in their altered expression of activin and TGFB-signaling transducers and antagonists. One seminoma tumor exhibited a particularly strong signal for both INHA and $\beta$-glycan, and a corresponding weak nuclear signal for phosphorylated SMAD2/3 (Fig. 5A-E), while the other two tumors demonstrated a comparatively weak signal for INHA and $\beta$-glycan, and a very strong signal for phosphorylated SMAD2/3 in the nucleus of seminoma cells (one of these tumors depicted in Fig. 5F-J). All three tumors demonstrated a strong SMAD6 signal and a weak to absent MAN-1 signal in seminoma cells (Fig. 5B, G and $\mathrm{C}, \mathrm{H}$ depicts SMAD6 and MAN-1 localization respectively in two of these three tumors). These results suggest there are distinct differences in the response of these three tumors to activin and TGFB signaling. Within the morphologically heterogeneous nonseminoma samples, phosphorylated SMAD2/3,

\footnotetext{
Figure 3 Cellular localization of activin and TGFB-signaling modulator proteins within gonadotropin-deprived human testis. Immunohistochemistry on testis sections from untreated men (A-E) and normal men that had received hormone treatment for 12 weeks with testosterone (F-J) for phosphorylated SMAD2/3 (A and F), SMAD6 (B and $G$ ), MAN-1 (C and H), INHA (D and I), and $\beta$-glycan (E and J). In untreated and gonadotropin-deprived samples, phosphorylated SMAD2/3 signal ( $A$ and F) localized to the nucleus of Sertoli cells and all germ cell types, and the signal is relatively stronger in the 12-week testosterone-treated samples. SMAD6 staining ( $B$ and $G$ ) was present within the nucleus and cytoplasm of Sertoli cells, spermatogonia, and spermatocytes. The SMAD6 signal was stronger in the spermatocyte cytoplasm in 12-week testosterone-treated samples relative to untreated sections (panel G left-hand insert indicates high magnification of spermatocyte cytoplasmic signal). MAN-1 protein $(\mathrm{C}$ and $\mathrm{H})$ was detected in the nuclear periphery and cytoplasm of spermatogonia, Sertoli cells, and occasional spermatocytes within untreated and gonadotropin-deprived samples (panel $\mathrm{H}$ left-hand insert indicates high magnification of stained spermatogonium). INHA (D and I) and $\beta$-glycan ( $E$ and $J)$ signals were detected in association with Sertoli cells in untreated and 12-week testosterone-treated samples, while $\beta$-glycan protein also localized to round spermatids in 12-week testosteronetreated sections (panel J left-hand insert indicates high magnification of round spermatid). Right-hand panels represent negative control sections lacking each primary antibody. White arrow, spermatogonial cell staining; hatched arrowhead, spermatocyte staining; white arrowhead, round spermatid staining; black arrow, Sertoli cell staining. Testosterone; pSMAD2/3, phosphorylated SMAD2/3. Scale bar in panel $\mathrm{A}$ indicates $50 \mu \mathrm{m}$ and is representative of all other panels.
} 
SMAD6, MAN-1, INHA, and $\beta$-glycan were detected in association with a variety of cell types (Fig. $5 \mathrm{~K}-\mathrm{O}$ and Table 1). Specifically, phosphorylated SMAD2/3 exhibited minimal to absent immunostaining in the embryonal carcinoma component, but demonstrated positive staining in stromal fibroblasts/fibrocyte-like cells and in a subset of cells in the somatically differentiated component. Anti-SMAD6 protein showed weak staining in the embryonal carcinoma cells (Fig. 5L) and strong staining in a subset of cells within glandular epithelium, and in some large cells resembling adipocytes. The inner nuclear membrane protein MAN-1 localized to scattered round mesenchymal-like cells. This staining was also moderate in embryonal carcinoma cells and was

Normal tubules in CIS
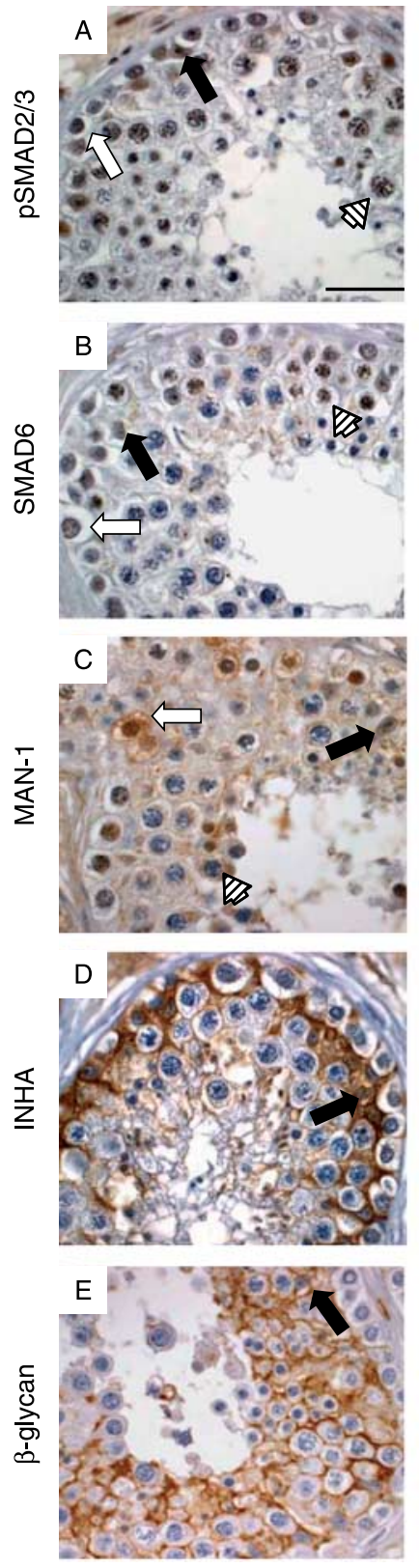

CIS
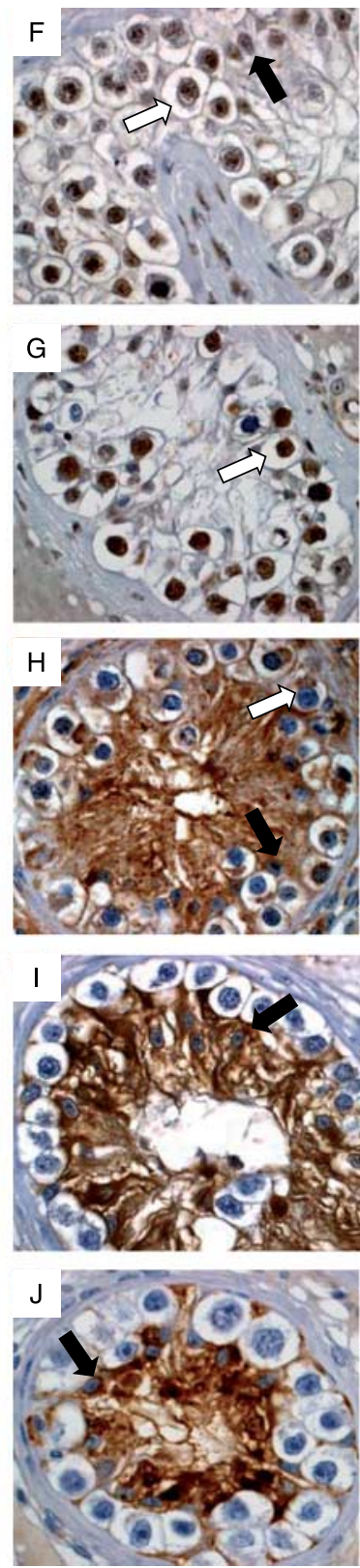

heterogeneously positive in glandular epithelial cells. Immunostaining for INHA was predominantly detectable in stromal fibroblasts/fibrocyte-like cells and in round lymphocyte-resembling cells, with heterogeneous staining in glandular epithelium and weak staining in a subset of embryonal carcinoma cells. Strong immunostaining for $\beta$-glycan was evident in squamous epithelium and vascular endothelium, including lymphatic vessels, and in a subset of glandular epithelium, while moderate staining was detected in the embryonal carcinoma component in a subset of samples (Fig. 5O). These findings demonstrate expression of activin/TGFB-signaling modulators persists in the testis following transformation of normal germ cells to the malignant nongerm cell phenotype of nonseminomas.

\section{Discussion}

This study has defined for the first time the cellular production sites of several activin and TGFB-signaling modulators within the normal adult human testis seminiferous epithelium and in its dysfunctional and neoplastic counterparts. Common sites of expression identify Sertoli cells and spermatogonia as the predominant cellular sites of activin/TGFB signal transduction and regulation (Fig. 6). These results are in accordance with our previous findings, which identify activin receptors in spermatogonia and Sertoli cells of the normal adult human testis (Dias et al. 2008). Our findings also identify a subset of tumors in which expression of activin/TGFB transducers and antagonists is distinctly altered in a trend that correlates with either increased or decreased signaling. Comparison of normal and gonadotropin-deprived human testis samples demonstrate changes in the cellular localization and expression patterns of activin/TGFB modulators, indicating that activin and TGFB-signaling regulation is sensitive to fluctuations in testis hormone levels.

Figure 4 Cellular localization of activin and TGFB-signaling modulator proteins within CIS specimens. Immunohistochemistry on testis sections from CIS patients with some tubules containing preserved spermatogenesis $(\mathrm{A}-\mathrm{E})$, and patients with only CIS tubules $(\mathrm{F}-\mathrm{J})$ for phosphorylated SMAD2/3 (A and F), SMAD6 (B and G), MAN-1 $(\mathrm{C}$ and $\mathrm{H})$, INHA (D and I), and $\beta$-glycan (E and J). Within tubules containing ongoing spermatogenesis in CIS samples, phosphorylated SMAD2/3 signals (A) were evident within the nucleus of Sertoli cells and all germ cell types; SMAD6 (B) within the nucleus of Sertoli cells, spermatogonia, and spermatocytes; MAN-1 (C) within the nucleus and cytoplasm of Sertoli cells, spermatogonia, and some spermatocytes and rounds; and INHA (D) and $\beta$-glycan (E) in association with Sertoli cells. In CIS tubules, phosphorylated SMAD2/3 (F) and SMAD6 (G) signals were present in the nucleus of CIS cells; MAN-1 $(\mathrm{H})$ in the cytoplasm of CIS cells, while INHA (I), and $\beta$-glycan (J) proteins were not detected in CIS cells. White arrow, spermatogonium or CIS cell staining; hatched arrowhead, spermatocyte staining; black arrow, Sertoli cell staining. CIS, carcinoma in situ; pSMAD2/3, phosphorylated SMAD2/3. Scale bar in panel $\mathrm{A}$ indicates $50 \mu \mathrm{m}$ and is representative of all other panels. 

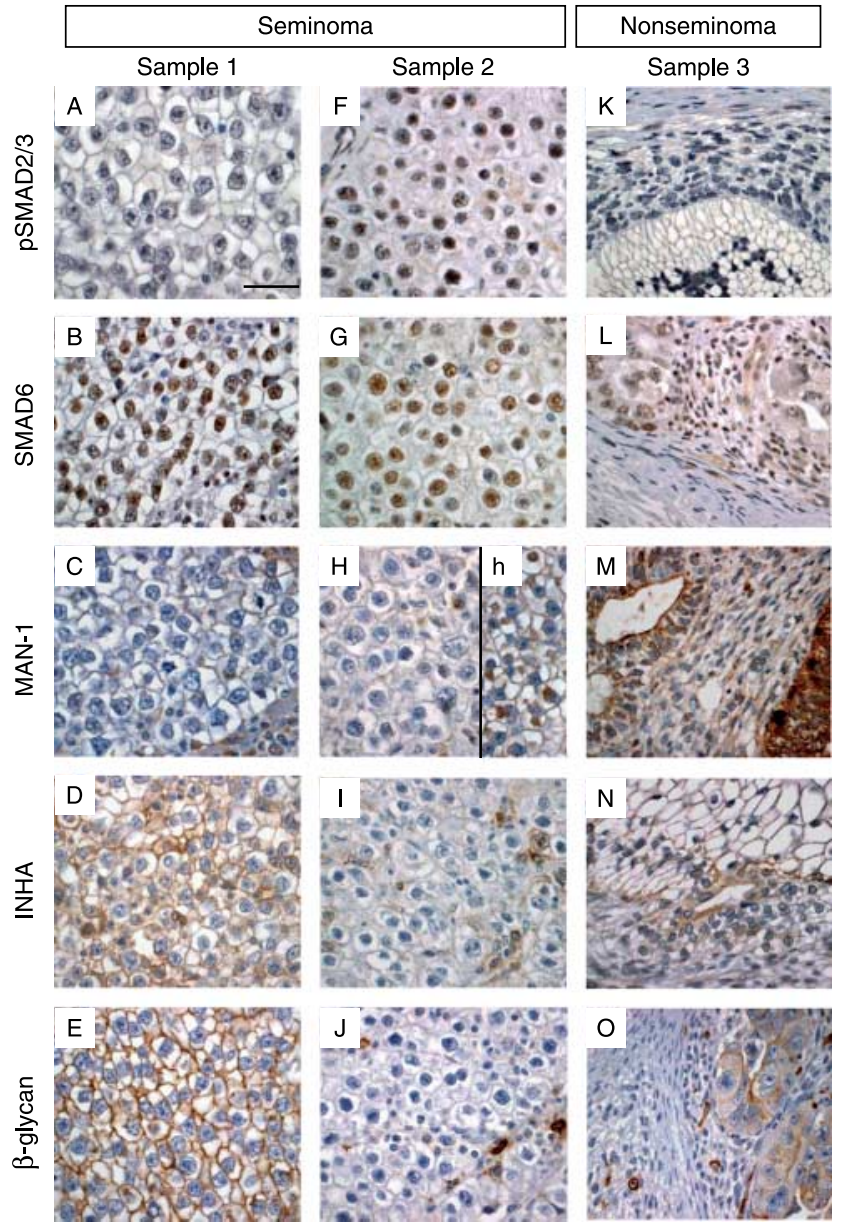

Figure 5 Cellular localization of activin and TGFB-signaling modulator proteins within testicular neoplasms. Immunohistochemistry on two seminoma tumors that exhibited distinct correlations in their altered expression of activin and TGFB-signaling modulator proteins (A-J), a third seminoma tumor that demonstrated an enhanced MAN-1 signal (h), and on nonseminoma tumors (K-O) for phosphorylated SMAD2/3 (A, F and K), SMAD6 (B, G and L), MAN-1 (C, H, h and M), INHA (D, I and $N$ ), and $\beta$-glycan ( $E, J$ and $O$ ). One seminoma tumor (A-E) displayed a strong INHA (D) and $\beta$-glycan (E) signal and a corresponding weak phosphorylated SMAD2/3 (A) signal. A second seminoma tumor (F-J) displayed a comparatively weak INHA (I) and $\beta$-glycan (J) signal and a strong phosphorylated SMAD2/3 (F) signal. Both tumors demonstrated a strong SMAD6 (B and G) signal and a weak to absent MAN-1 ( $\mathrm{C}$ and $\mathrm{H}$ ) signal in seminoma cells. An example of a third seminoma tumor that exhibited a comparatively strong signal across majority of the section for MAN-1 (h). In the heterogeneous nonseminoma tumors $(\mathrm{K}-\mathrm{O})$, all antigens were detected within a variety of cell types. pSMAD2/3, phosphorylated SMAD2/3. Scale bar in panel $A$ indicates $50 \mu \mathrm{m}$ and is representative of all other panels.

The activin/TGFB-signaling transducers phosphorylated SMAD2/3, and antagonists, MAN-1, INHA and $\beta$-glycan, exhibited heterogeneous expression patterns within and between seminoma tumors. INHA and $\beta$-glycan are normally produced by somatic cells in the adult testis; their synthesis in a subset of germ cellderived seminomas may allow selected tumors to gain independence from Sertoli cell-derived factors as they gain the capacity to antagonize activin signaling in an autocrine manner and thereby limit activin bioactivity. Our data illustrating a differential expression of the INHA subunit between individual seminoma specimens ( 6 out of 28) is consistent with a previous report documenting the presence of INHA mRNA in a subset of seminomas (three out of nine) using RNAase protection assays (van Schaik et al. 1997). In the normal testis, production of the INHA subunit is confined to Sertoli and Leydig cells in species, including the human and mouse (Roberts 1997, Majdic et al. 1997, Barakat et al. 2008). Mice lacking the INHA subunit develop Sertoli cell tumors 3-4 weeks after birth, due to excessive activin production and signaling (Matzuk et al. 1992). The absence of Sertoli cells in germ cell-derived tumors may also reflect excessive activin signaling, as the ActRIIA subunit is consistently up-regulated in seminomas (Dias et al. 2008). The synthesis of INHA by a subset of seminomas may therefore engender in cells in these tumors the capacity to suppress activin signaling, with implications for the growth of seminoma cells (Fig. 6).

In addition to inhibiting activin, $\beta$-glycan facilitates signaling by all three TGFB (TGFB1-B3) ligands and enhances BMP signaling by directly binding BMP2, 4, 7 and GDF5 to augment their binding to type I BMP receptors (Lopez-Casillas et al. 1993, Kirkbride et al. $2008)$. Therefore, the synthesis of $\beta$-glycan protein by a subset of seminomas would confer responsiveness to several additional TGFB superfamily signaling molecules. In other human cancers, such as breast, ovary, lung, pancreas, and prostate carcinomas, loss of $\beta$-glycan mRNA and protein has been linked to enhanced migration and invasion by tumor cells (Dong et al. 2007, Hempel et al. 2007, Turley et al. 2007, Finger et al. 2008, Gordon et al. 2008), indicating a tumor suppressive function of $\beta$-glycan. If $\beta$-glycan functions in a similar manner in TGCTs, its detection within particular specimens may reflect a tendency for these tumors to remain confined to their site of origin.

The integral inner nuclear membrane protein MAN-1 inhibits activin signaling by interfering with signal transduction via SMAD2/3 proteins and restricting transcriptional activation of activin/TGFB response genes (Pan et al. 2005, Lin et al. 2005, Caputo et al. 2006). Mutations in MAN-1 have been linked to a number of human diseases characterized by increased bone density, implicating MAN-1 as a negative regulator of bone formation by inhibiting TGFB superfamily signaling (Hellemans et al. 2004). To date, neither the expression nor function of MAN-1 in human neoplasms has been investigated. The presence of MAN-1 in both Sertoli and germ cells of the normal adult human testis indicates that it is directly involved in the function and/or development of both cell lineages. The enhanced MAN-1 protein signal detected in a subset of seminomas may reflect an additional level of TGFB superfamily signaling regulation that would lead to altered 


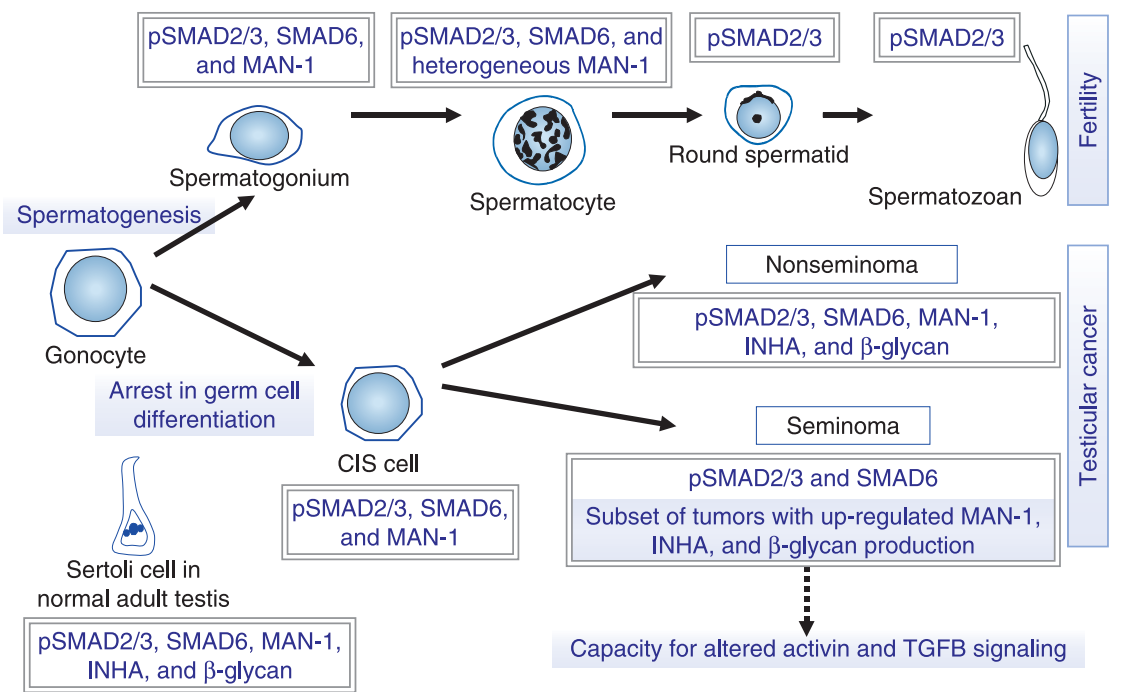

Figure 6 Schematic illustration of normal spermatogenesis, the pathogenesis of CIS, its progression to malignant neoplasms, and the cellular localization of activin/TGFB-signaling modulators within these. In the normal context, gonocytes undergo mitosis and differentiate into spermatogonia that progress through different stages of spermatogenesis to give rise to mature sperm (top). Within the normal adult testis, phosphorylated SMAD2/3 were expressed by all germ cell types, SMAD6 by spermatogonia and spermatocytes, MAN-1 by spermatogonia and occasional spermatocytes, while all tested signaling modulators were expressed by Sertoli cells. In the neoplastic state, an arrest in the differentiation program of fetal germ cells causes gonocytes to transform to premalignant CIS cells, which progress to malignant seminomas or nonseminomas after puberty (bottom). Phosphorylated SMAD2/3, SMAD6, and MAN-1 are produced by CIS cells. All seminoma tumors exhibited phosphorylated SMAD2/3 and SMAD6 production, while MAN-1, INHA, and $\beta$-glycan were up-regulated in a subset of seminomas, highlighting the capacity for changed activin/TGFB-signaling modulation to occur in response to spermatogenic disruption.

expression of activin/TGFB target genes in these testicular tumor cells (Fig. 6).

Changes in the expression and cellular localization of activin signaling regulators in the 12-week gonadotropindeprived testis sections suggest that testicular responsiveness to activin/TGFB stimulation is modulated by spermatogenic disruption and altered testicular hormone levels and highlight the potential of TGFB superfamily signaling to influence testicular physiology. The administration of testosterone or testosterone plus DMPA to healthy adult men causes significant impairment in the transition of type B spermatogonial cells to early spermatocytes and profound inhibition of sperm release (McLachlan et al. 2002). $\beta$-Glycan protein induction in haploid germ cells in gonadotropin-deprived testis would be predicted to enhance suppression of activin signaling and thereby increase TGFB and BMP ligand responsiveness. SMAD6 has been shown to preferentially antagonize BMP signaling (Ishisaki et al. 1999, Goto et al. 2007). The increased SMAD6 signal in these spermatocytes may reflect circumstances in which activin signaling is enhanced due to reduced competition with BMPs for signal transducing machinery. Collectively, the enhanced TGFB ligand signaling conferred by $\beta$-glycan synthesis in round spermatids and enhanced activin signaling caused by preferential SMAD6 inhibition of BMP signaling may account for the particularly strong nuclear phosphorylated SMAD2/3 signal within 12-week testosterone or testosterone plus DMPA samples. These results are in agreement with our previous findings of enhanced ActRIIA protein production within the gonadotropin-deprived testis that could allow for enhanced activin and TGFB ligand signaling (Dias et al. 2008).

The progression of CIS to malignancy is associated with puberty, as seen by a sharp rise in the age-specific incidence of germ cell tumors following puberty (Rajpert-De Meyts \& Skakkebaek 1993). The dramatic change in testicular hormone production is a likely trigger for this malignant transformation. The induction of $\beta$-glycan protein in a subset of samples displaying neoplastic transformation of germ cells and also following hormonal manipulation of the testis highlights the potential of $\beta$-glycan to either influence or be effected by spermatogenic disruption. We hypothesize further that $\beta$-glycan synthesis by germ cells is influenced by changes in testicular testosterone levels. Differences in $\beta$-glycan protein production between seminoma specimens may reflect differences in the hormonal environment between individual testis tumors.

The molecular events associated with CIS progression to malignancy remain largely unknown. Our results identify distinct differences between individual TGCTs in their potential response to and modulation of activin and TGFB signaling. The production of INHA and $\beta$-glycan by somatic cells in the human fetal testis (Majdic et al. 1997, Anderson et al. 2002) at a time when germ cells are sensitive to transformation to premalignant germ cells highlights the importance of regulated activin/TGFB signaling during fetal germ cell 
development. Therefore, the aberrant expression of somatically expressed activin antagonists by a subset of seminomas may lead to altered activin bioactivity and change germ cell tumor behavior (Fig. 6). Given the findings of this study, further investigations into the role of activin/TGFB signaling and their regulation in testicular tumor development and their potential effect on clinical outcomes are warranted.

\section{Materials and Methods}

\section{Samples}

Histological analysis was performed on Bouins fixed, paraffinembedded sections of normal adult human testis, TGCTs, and gonadotropin-deprived testes. Testicular cancer samples consisting of CIS with or without areas of morphologically normal spermatogenesis, seminomas, and nonseminomas were obtained from pathology departments in the greater Copenhagen area. All tumor samples were assessed by experienced pathologists and routinely stained with placental like alkaline phosphatase (PLAP) to identify CIS cells. Permission for their collection and use in immunohistochemical studies was obtained from the Regional Committee for Medical Research Ethics in Denmark.

All other sections were obtained from biopsy samples collected from healthy adult volunteers. Samples of gonadotropin-deprived testis were obtained following treatment with either testosterone or testosterone plus a depot progestin (testosterone+DMPA) for 12 weeks samples from McLachlan et al. (2002). Testes samples from control men not subjected to testosterone or testosterone+DMPA treatment were also analyzed. Sample collection was approved by the Human Research and Ethics Committee of the Monash Medical Centre, and informed consent was obtained from each subject. The numbers of normal, neoplastic, and gonadotropindeprived testes samples examined are listed in Table 1.

\section{Immunohistochemistry}

Immunohistochemistry was performed as previously described on dewaxed sections blocked with $5 \%(\mathrm{v} / \mathrm{v})$ normal serum diluted in Tris-buffered saline (TBS) with $0.1 \%$ BSA before the addition of primary antibody (Loveland et al. 1999). Briefly, antigen retrieval was performed at $90^{\circ} \mathrm{C}$ and maintained for 8 mins in 50 mM glycine pH 3.5 for phosphorylated SMAD2/3, SMAD6 and INHA, $0.01 \mathrm{M}$ Citrate $\mathrm{pH} 6.0$ for $\beta$-glycan, and testosterone-EG (10 mM Tris, $0.5 \mathrm{mM}$ EGTA) pH 9.0 for MAN-1. Samples were blocked in $5 \%(\mathrm{v} / \mathrm{v})$ normal serum (normal sheep serum for phosphorylated SMAD2/3, SMAD6, and INHA and normal rabbit serum for MAN-1 and $\beta$-glycan), diluted in TBS with $0.1 \%$ BSA for $1 \mathrm{~h}$. Primary antibodies were then applied: phosphorylated SMAD2/3 at 1:150 dilution, SMAD6 at $1.7 \mu \mathrm{g} / \mathrm{ml}, \mathrm{MAN}-1$ at $2.7 \mu \mathrm{g} / \mathrm{ml}$, INHA at $65.5 \mu \mathrm{g} /$ $\mathrm{ml}$, and $\beta$-glycan at $1.25 \mu \mathrm{g} / \mathrm{ml}$, diluted in TBS with $0.1 \%$ BSA for overnight incubation at room temperature. All washes between incubations were performed at room temperature with TBS. Primary antibody binding was detected using a biotinylated secondary antibody: sheep anti-rabbit (Chemicon,
Temecula, CA, USA) for phosphorylated SMAD2/3 and SMAD6, rabbit anti-goat (DAKO, Botany, NSW, Australia) for MAN- 1 and $\beta$-glycan, and sheep anti-mouse (Chemicon) for INHA (each diluted 1:500 in TBS/0.1\% BSA, $1 \mathrm{~h}$ ). The Vectastain Elite $A B C$ kit was then applied according to the manufacturer's instructions (Vector Laboratories, Burlingame, CA, USA). Antibody binding was detected as a brown precipitate following development with 3,3'-diaminobenzidine tetrahydrochloride against a Harris hematoxylin counterstain. Germ cell types were identified primarily on the basis of their nuclear morphology and position within the seminiferous epithelium, as described in McLachlan et al. (2002). Immunohistochemistry was performed at least in duplicate with identical results between experiments for each sample. The number of individual samples tested from each treatment or sample group for each antibody is listed in Table 1. Immunostained sections were scored blinded by relative staining intensity as high, medium, low, and negative by subjective semiquantitative analysis. To account for variations in staining intensity between different immunohistochemistry runs, adult human testis samples were included in each run; these consistently demonstrated identical results. Controls for nonspecific binding of the secondary antibody were performed in all experiments by omission of primary antibody, and these consistently yielded no signal within the seminiferous epithelium or in the interstitial space.

\section{Antibody validation and western blot analyzes}

Selective target recognition by the mouse monoclonal INHA R1 antibody (Groome N) has been previously established (Groome \& O'Brien 1993, Arora et al. 1997). Western blot validation of the rabbit polyclonal anti-phospho-SMAD2/3 (Cell Signaling Technology, Danvers, MA, USA) antibody was performed on immature day 6 mouse Sertoli cell lysates (the amino acid sequences of both SMAD2 and SMAD3 proteins share $>95 \%$ sequence homology between mouse and human), and yielded a single band of the expected size of $58 \mathrm{kDa}$ (Itman et al. 2009). Manufacturer's specifications indicate this antibody will detect both phosphorylated SMAD2 and SMAD3 in fixed tissues due to sequence similarity between these two proteins. The rabbit polyclonal anti-SMAD6 antibody (Zymed Laboratories, San Franciso, CA, USA) has also been used on western blots of mouse testis lysates to detect a single band of $60 \mathrm{kDa}$ (Itman \& Loveland 2008; amino acid sequences of human and mouse SMAD6 share $~ 95 \%$ sequence homology). An individual sample of the goat polyclonal anti-human TGFBR3 antibody (R\&D Systems, Minneapolis, MN, USA) was previously verified by immunofluorescence analysis on a $\beta$-glycan null mouse line (Sarraj et al. 2007; amino acid sequence of human and mouse $\beta$-glycan shares $\sim 90 \%$ sequence homology).

Target recognition by the goat polyclonal anti-MAN-1 (N-20; Santa Cruz Biotechnology) antibody was determined by western blot analysis on human NTERA cell lysates. Protein loading was indicated by blotting for $\alpha$-tubulin (Sigma Chemical Co). Briefly, lysates were prepared by homogenization at $4{ }^{\circ} \mathrm{C}$ in RIPA buffer $(150 \mathrm{mM}$ sodium chloride, $1 \%$ Nonidet P-40, $0.5 \%$ Tween-20, $0.1 \%$ SDS, $1 \mathrm{mM}$ 
ethylenediaminetetraacetic acid) in the presence of protease inhibitors (Protease Inhibitor Cocktail Set III, Calbiochem, San Diego, CA, USA), as previously described (Loveland et al. 1999). Protein concentration was determined using the BioRad DC protein assay (Bio-Rad). A total of $30 \mu \mathrm{g}$ of human NTERA cell protein lysate was loaded per lane onto $12 \%$ SDS-PAGE gels with protein size standards (Page-Ruler Prestained Protein Ladder, Fermentas, Burlington, Ontario, Canada). Following electrophoresis, the proteins were transferred to Hybond-C Nitrocellulose membrane (Amersham Biosciences) and all subsequent incubations were performed at room temperature unless otherwise stated. The membranes were blocked for $1 \mathrm{~h}$ with Odyssey buffer:PBS (1:2) (LICOR Biosciences, Lincoln, NE, USA). Primary antibody incubations were carried out at $4{ }^{\circ} \mathrm{C}$ in blocking buffer plus $0.1 \%(\mathrm{v} / \mathrm{v})$ Tween. MAN- 1 and $\alpha$-tubulin antibodies were used at dilutions or concentrations of $0.6 \mu \mathrm{g} / \mathrm{ml}$ and 1:5000 respectively. Membranes were washed with PBS plus $0.1 \%$ Tween $(\mathrm{v} / \mathrm{v})$, then incubated with Alexa Fluor 680-coupled secondary antibodies (Molecular Probes, Eugene, OR, USA) at 1:10 000 dilution in blocking solution with $0.1 \%(\mathrm{v} / \mathrm{v})$ Tween and $0.01 \%(\mathrm{v} / \mathrm{v})$ SDS for $1 \mathrm{~h}$. Bound antibody was detected using the LI-COR Odyssey System (LI-COR Biosciences).

\section{Declaration of interest}

The authors declare that there is no conflict of interest that could be perceived as prejudicing the impartiality of the research reported.

\section{Funding}

This work was supported by the National Health and Medical Research Council of Australia (Grants \#545916 and \#545917 to K Loveland and \#169020 to R McLachlan), the Danish Cancer Society (Grant \# DP05113 to E Rajpert-De Meyts), the Australian Research Council (\#348239 to K Loveland), and Monash University (Postgraduate Scholarship to V Dias).

\section{References}

Anderson RA, Cambray N, Hartley PS \& McNeilly AS 2002 Expression and localization of inhibin alpha, inhibin/activin betaA and betaB and the activin type II and inhibin beta-glycan receptors in the developing human testis. Reproduction 123 779-788.

Andersson AM, Muller J \& Skakkebaek NE 1998 Different roles of prepubertal and postpubertal germ cells and Sertoli cells in the regulation of serum inhibin B levels. Journal of Clinical Endocrinology and Metabolism 83 4451-4458.

Arora DS, Cooke IE, Ganesan TS, Ramsdale J, Manek S, Charnock FM, Groome NP \& Wells M 1997 Immunohistochemical expression of inhibin/activin subunits in epithelial and granulosa cell tumours of the ovary. Journal of Pathology 181 413-418.

Bai S \& Cao X 2002 A nuclear antagonistic mechanism of inhibitory Smads in transforming growth factor-beta signaling. Journal of Biological Chemistry 277 4176-4182.

Barakat B, O'Connor A, Gold E, de Kretser D \& Loveland K 2008 Inhibin, activin, follistatin and follicle stimulating hormone serum levels and testicular production are highly modulated during the first spermatogenic wave in mice. Reproduction 136 345-359.
Bergstrom R, Adami HO, Mohner M, Zatonski W, Storm H, Ekbom A, Tretli S, Teppo L, Akre O \& Hakulinen T 1996 Increase in testicular cancer incidence in six European countries: a birth cohort phenomenon. Journal of National Cancer Institute 88 727-733.

Boitani C, Stefanini M, Fragale A \& Morena AR 1995 Activin stimulates Sertoli cell proliferation in a defined period of rat testis development. Endocrinology 136 5438-5444.

Brown CW, Houston-Hawkins DE, Woodruff TK \& Matzuk MM 2000 Insertion of Inhbb into the Inhba locus rescues the Inhba-null phenotype and reveals new activin functions. Nature Genetics 25 453-457.

Caputo S, Couprie J, Duband-Goulet I, Konde E, Lin F, Braud S, Gondry M, Gilquin B, Worman HJ \& Zinn-Justin S 2006 The carboxyl-terminal nucleoplasmic region of MAN1 exhibits a DNA binding winged helix domain. Journal of Biological Chemistry 281 18208-18215.

Chang H, Brown CW \& Matzuk MM 2002 Genetic analysis of the mammalian transforming growth factor-beta superfamily. Endocrine Reviews 23 787-823.

Cobellis L, Cataldi P, Reis FM, De Palo G, Raspagliesi F, Pilotti S, Arcuri F \& Petraglia F 2001 Gonadal malignant germ cell tumors express immunoreactive inhibin/activin subunits. European Journal of Endocrinology 145 779-784.

Denissova NG, Pouponnot C, Long J, He D \& Liu F 2000 Transforming growth factor beta-inducible independent binding of SMAD to the Smad7 promoter. PNAS 97 6397-6402.

Dias V, Meachem S, Rajpert-De Meyts E, McLachlan R, Manuelpillai U \& Loveland KL 2008 Activin receptor subunits in normal and dysfunctional adult human testis. Human Reproduction 23 412-420.

Dong M, How T, Kirkbride KC, Gordon KJ, Lee JD, Hempel N, Kelly P, Moeller BJ, Marks JR \& Blobe GC 2007 The type III TGF-beta receptor suppresses breast cancer progression. Journal of Clinical Investigation 117 206-217.

Esparza-Lopez J, Montiel JL, Vilchis-Landeros MM, Okadome T, Miyazono K \& Lopez-Casillas F 2001 Ligand binding and functional properties of betaglycan, a co-receptor of the transforming growth factorbeta superfamily. Specialized binding regions for transforming growth factor-beta and inhibin A. Journal of Biological Chemistry 276 14588-14596.

Finger EC, Turley RS, Dong M, How T, Fields TA \& Blobe GC 2008 TbetaRIII suppresses non-small cell lung cancer invasiveness and tumorigenicity. Carcinogenesis 29 528-535.

Fragale A, Puglisi R, Morena AR, Stefanini M \& Boitani C 2001 Age-dependent activin receptor expression pinpoints activin $\mathrm{A}$ as a physiological regulator of rat Sertoli cell proliferation. Molecular Human Reproduction 7 1107-1114.

Gordon KJ, Dong M, Chislock EM, Fields TA \& Blobe GC 2008 Loss of type III transforming growth factor beta receptor expression increases motility and invasiveness associated with epithelial to mesenchymal transition during pancreatic cancer progression. Carcinogenesis 29 252-262.

Goto K, Kamiya Y, Imamura T, Miyazono K \& Miyazawa K 2007 Selective inhibitory effects of Smad6 on bone morphogenetic protein type I receptors. Journal of Biological Chemistry 282 20603-20611.

Groome N \& O'Brien M 1993 Immunoassays for inhibin and its subunits. Further applications of the synthetic peptide approach. Journal of Immunological Methods 165 167-176.

Hanyu A, Ishidou Y, Ebisawa T, Shimanuki T, Imamura T \& Miyazono K 2001 The $N$ domain of Smad7 is essential for specific inhibition of transforming growth factor-beta signaling. Journal of Cell Biology 155 1017-1027.

Harrison CA, Wiater E, Gray PC, Greenwald J, Choe S \& Vale W 2004 Modulation of activin and BMP signaling. Molecular and Cellular Endocrinology 225 19-24.

Harrison CA, Gray PC, Vale WW \& Robertson DM 2005 Antagonists of activin signaling: mechanisms and potential biological applications. Trends in Endocrinology and Metabolism 16 73-78.

Hellemans J, Preobrazhenska O, Willaert A, Debeer P, Verdonk PC, Costa T, Janssens K, Menten B, Van Roy N, Vermeulen SJ et al. 2004 Loss-of-function mutations in LEMD3 result in osteopoikilosis, BuschkeOllendorff syndrome and melorheostosis. Nature Genetics 36 1213-1218.

Hempel N, How T, Dong M, Murphy SK, Fields TA \& Blobe GC 2007 Loss of betaglycan expression in ovarian cancer: role in motility and invasion. Cancer Research 67 5231-5238. 
Imamura T, Takase M, Nishihara A, Oeda E, Hanai J, Kawabata M \& Miyazono K 1997 Smad6 inhibits signaling by the TGF-beta superfamily. Nature 389 622-626.

Ishida W, Hamamoto T, Kusanagi K, Yagi K, Kawabata M, Takehara K, Sampath TK, Kato M \& Miyazono K 2000 Smad6 is a Smad1/5-induced smad inhibitor. Characterization of bone morphogenetic proteinresponsive element in the mouse Smad6 promoter. Journal of Biological Chemistry 275 6075-6079.

Ishisaki A, Yamato K, Hashimoto S, Nakao A, Tamaki K, Nonaka K, ten Dijke P, Sugino H \& Nishihara T 1999 Differential inhibition of Smad6 and Smad7 on bone morphogenetic protein- and activinmediated growth arrest and apoptosis in B cells. Journal of Biological Chemistry 274 13637-13642.

Itman C \& Loveland KL 2008 SMAD expression in the testis: an insight into BMP regulation of spermatogenesis. Developmental Dynamics 237 97-111.

Itman C, Mendis S, Barakat B \& Loveland KL 2006 All in the family: TGFbeta family action in testis development. Reproduction 132 233-246.

Itman C, Whiley PA, Zhou W, Meistrich M, Sahin Z \& Loveland KL 2009 Regulated production of $\mathrm{SnoN} 2$ is a feature of testicular differentiation. Microscopy Research and Technique [in press]. DOI: 10.1002/ jemt.20739.

Itoh S, Landstrom M, Hermansson A, Itoh $\mathrm{F}$, Heldin $\mathrm{CH}$, Heldin NE \& ten Dijke $\mathbf{P} 1998$ Transforming growth factor beta1 induces nuclear export of inhibitory Smad7. Journal of Biological Chemistry 273 29195-29201.

Kirkbride KC, Townsend TA, Bruinsma MW, Barnett JV \& Blobe GC 2008 Bone morphogenetic proteins signal through the transforming growth factor-\{beta\} type III receptor. Journal of Biological Chemistry 283 7628-7637.

Konrad L, Keilani MM, Laible L, Nottelmann U \& Hofmann R 2006 Effects of TGF-betas and a specific antagonist on apoptosis of immature rat male germ cells in vitro. Apoptosis 11 739-748.

Lewis KA, Gray PC, Blount AL, MacConell LA, Wiater E, Bilezikjian LM \& Vale W 2000 Betaglycan binds inhibin and can mediate functional antagonism of activin signaling. Nature 404 411-414.

Lin F, Morrison JM, Wu W \& Worman HJ 2005 MAN1, an integral protein of the inner nuclear membrane, binds Smad2 and Smad3 and antagonizes transforming growth factor-beta signaling. Human Molecular Genetics 14 437-445.

Lin SJ, Lerch TF, Cook RW, Jardetzky TS \& Woodruff TK 2006 The structural basis of TGF-beta, bone morphogenetic protein, and activin ligand binding. Reproduction 132 179-190.

Lopez-Casillas F, Wrana JL \& Massague J 1993 Betaglycan presents ligand to the TGF beta signaling receptor. Cell 73 1435-1444.

Loveland KL \& Robertson DM 2005 The TGF $\beta$ Superfamily in Sertoli Cell Biology. San Diego, CA, USA: Elsevier Science.

Loveland KL, Herszfeld D, Chu B, Rames E, Christy E, Briggs LJ, Shakri R, de Kretser DM \& Jans DA 1999 Novel low molecular weight microtubuleassociated protein-2 isoforms contain a functional nuclear localization sequence. Journal of Biological Chemistry 274 19261-19268.

Loveland KL, Bakker M, Meehan T, Christy E, von Schonfeldt V, Drummond A \& de Kretser D 2003 Expression of Bambi is widespread in juvenile and adult rat tissues and is regulated in male germ cells. Endocrinology 144 4180-4186.

Majdic G, McNeilly AS, Sharpe RM, Evans LR, Groome NP \& Saunders PT 1997 Testicular expression of inhibin and activin subunits and follistatin in the rat and human fetus and neonate and during postnatal development in the rat. Endocrinology 138 2136-2147.

Marchetti C, Hamdane M, Mitchell V, Mayo K, Devisme L, Rigot JM, Beauvillain JC, Hermand E \& Defossez A 2003 Immunolocalization of inhibin and activin alpha and betaB subunits and expression of corresponding messenger RNAs in the human adult testis. Biology of Reproduction 68 230-235.

Mather JP, Attie KM, Woodruff TK, Rice GC \& Phillips DM 1990 Activin stimulates spermatogonial proliferation in germ-Sertoli cell cocultures from immature rat testis. Endocrinology 127 3206-3214.

Matzuk MM, Finegold MJ, Su JG, Hsueh AJ \& Bradley A 1992 Alpha-inhibin is a tumour-suppressor gene with gonadal specificity in mice. Nature $360313-319$.
Matzuk MM, Kumar TR, Vassalli A, Bickenbach JR, Roop DR, Jaenisch R \& Bradley A 1995 Functional analysis of activins during mammalian development. Nature 374 354-356.

Mazerbourg S, Sangkuhl K, Luo CW, Sudo S, Klein C \& Hsueh AJ 2005 Identification of receptors and signaling pathways for orphan bone morphogenetic protein/growth differentiation factor ligands based on genomic analyses. Journal of Biological Chemistry 280 32122-32132.

McLachlan RI, O'Donnell L, Stanton PG, Balourdos G, Frydenberg M, de Kretser DM \& Robertson DM 2002 Effects of testosterone plus medroxyprogesterone acetate on semen quality, reproductive hormones, and germ cell populations in normal young men. Journal of Clinical Endocrinology and Metabolism 87 546-556.

Meehan T, Schlatt S, O'Bryan MK, de Kretser DM \& Loveland KL 2000 Regulation of germ cell and Sertoli cell development by activin, follistatin, and FSH. Developmental Biology 220 225-237.

Nagarajan RP, Zhang J, Li W \& Chen Y 1999 Regulation of Smad7 promoter by direct association with Smad3 and Smad4. Journal of Biological Chemistry 274 33412-33418.

Olaso R, Pairault C, Boulogne B, Durand P \& Habert R 1998 Transforming growth factor beta1 and beta2 reduce the number of gonocytes by increasing apoptosis. Endocrinology 139 733-740.

Pan D, Estevez-Salmeron LD, Stroschein SL, Zhu X, He J, Zhou S \& Luo K 2005 The integral inner nuclear membrane protein MAN1 physically interacts with the R-Smad proteins to repress signaling by the transforming growth factor-\{beta\} superfamily of cytokines. Journal of Biological Chemistry 280 15992-16001.

Rajpert-de Meyts E \& Hoei-Hansen CE 2007 From gonocytes to testicular cancer: the role of impaired gonadal development. Annals of the New York Academy of Sciences 1120 168-180.

Rajpert-De Meyts E \& Skakkebaek NE 1993 The possible role of sex hormones in the development of testicular cancer. European Urology $\mathbf{2 3}$ 54-59 discussion 60-51.

Roberts VJ 1997 Tissue-specific expression of inhibin/activin subunit and follistatin mRNAs in mid- to late-gestational age human fetal testis and epididymis. Endocrine 6 85-90.

Sarraj MA, Chua HK, Umbers A, Loveland KL, Findlay JK \& Stenvers KL 2007 Differential expression of TGFBR3 (betaglycan) in mouse ovary and testis during gonadogenesis. Growth Factors 25 334-345.

van Schaik RH, Wierikx CD, Looijenga LH, Oosterhuis JW \& de Jong FH 1997 Human testicular germ cell tumours express inhibin subunits, activin receptors and follistatin mRNAs. British Journal of Cancer $\mathbf{7 6}$ 1191-1198.

Shi Y \& Massague J 2003 Mechanisms of TGF-beta signaling from cell membrane to the nucleus. Cell 113 685-700.

Skakkebaek NE 1972 Possible carcinoma-in-situ of the testis. Lancet 2 516-517.

Sonne SB, Hoei-Hansen CE, Nielsen JE, Herlihy AS, Andersson AM, Almstrup K, Daugaard G, Skakkebaek NE, Leffers H \& Rajpert-De Meyts E 2006 CDH1 (E-cadherin) in testicular germ cell neoplasia: suppressed translation of mRNA in pre-invasive carcinoma in situ but increased protein levels in advanced tumours. Acta Pathologica, Microbiologica, et Immunologica Scandinavica 114 549-558.

Turley RS, Finger EC, Hempel N, How T, Fields TA \& Blobe GC 2007 The type III transforming growth factor-beta receptor as a novel tumor suppressor gene in prostate cancer. Cancer Research 67 1090-1098.

Vliegen MK, Schlatt S, Weinbauer GF, Bergmann M, Groome NP \& Nieschlag E 1993 Localization of inhibin/activin subunits in the testis of adult nonhuman primates and men. Cell Tissue Research 273 261-268.

Received 21 May 2009

First decision 19 June 2009

Revised manuscript received 26 July 2009

Accepted 6 August 2009 